\title{
Towards Shoestring Solutions for UK Manufacturing SMEs
}

\author{
Gregory Hawkridge Benjamin Schönfuß Duncan McFarlane Lavindra de Silva \\ German Terrazas Liz Salter Alan Thorne \\ Institute for Manufacturing (IfM), University of Cambridge, UK \\ gth24@cam.ac.uk
}
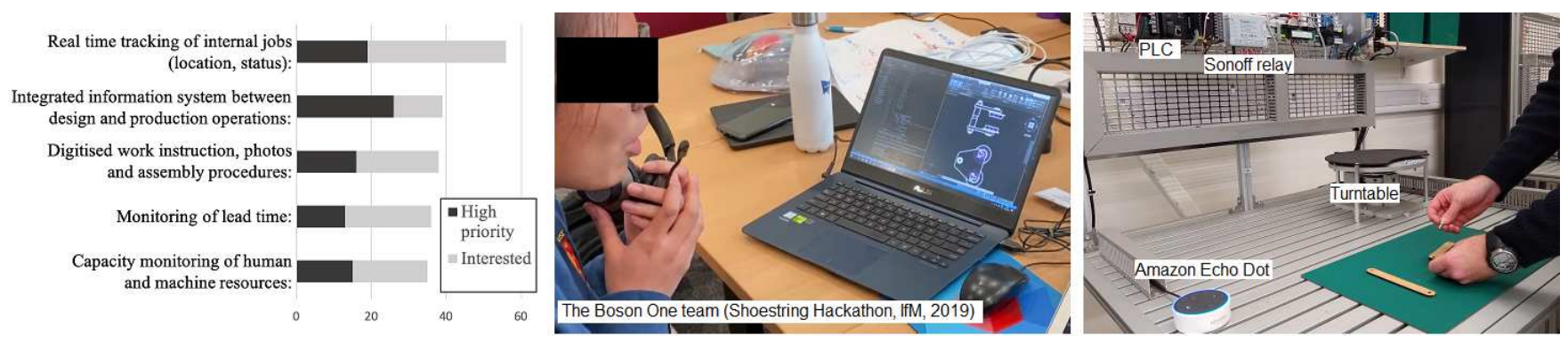

Figure 1: Top 5 solution areas (updated from [1] using recent workshop data) (left). Voice commanded digital drawing navigation using Google Speech-to-Text recognition (middle) and voice commanded turntable using Amazon Echo Dot (right).

\begin{abstract}
In the Digital Manufacturing on a Shoestring project we focus on low-cost digital solution requirements for UK manufacturing SMEs. This paper shows that many of these fall in the HRI domain while presenting the use of low-cost and off-the-shelf technologies in two demonstrators based on voice assisted production.
\end{abstract}

\section{KEYWORDS}

Voice assisted production; low cost demonstrators; UK SME needs

Our overall aim is to assess the suitability of low-cost, off-the-shelf and non-industrial digital technologies for manufacturing SMEs, who are often less able to adopt digital solutions compared to larger companies, due to high investment costs and a lack of skills. To this end, our methodology involves (1) investigating and prioritising the digital solutions that such SMEs need most, (2) gathering and classifying the available technologies, (3) building physical lab demonstrators and industrial pilots that realise the solutions, and (4) assessing their viability with input from industry and academia.

To study the digital needs of (manufacturing) SMEs [1], we derived an initial list of solutions requirements identified across 500 UK SME consultation activities. The list was further augmented with data from SME site visits, where we identified planned or adopted solutions. The prioritisation of the solution areas involved running workshops with groups up to 30 UK SMEs where each participant focused on segments that met their top two business needs and marked up to 3 "high priority" requirements and up to 6 "lower

Accepted to HRI 2020, The ACM/IEEE International Conference on Human-Robot Interaction, Cambridge, United Kingdom

(c) 2020 Copyright held by the owner/author(s)

This is the author's version of the work. It is posted here for your personal use. Not for redistribution. The definitive Version of Record was published in Companion of the 2020 ACM/IEEE International Conference on Human-Robot Interaction (HRI '20 Companion), March 23-26, 2020, Cambridge, United Kingdom, https://doi.org/10.1145/ 3371382.3378205 . priority" requirements for each. So far about 200 UK SMEs have participated in our workshops; Fig. 1 left shows their top 5 solutions by a combined number of high and lower priority markings. We observed that most high priority solution areas did not involve fully automated control of robots/hardware by software, but instead assisting or enhancing human decision making, for example providing instructions for completing a particular operation. We also observed other lower priority solutions areas relevant to HRI, such as guidance on quality standards; generating a joint work schedule linked to human and robot resources; generating job routings and presenting these to operators for checking; supporting operators in optimising output quality by providing information on historical problems with product quality or by giving insight into product application; and anticipating workpiece or tool requirements in production and sending instructions either to the operator, or to an automated solution for the delivery of this equipment directly to the operator.

We have built two example solutions to demonstrate the industrial potential of low-cost, off-the-shelf and non-industrial technologies, e.g. for variants of the last solution above. The first one captures voice commands using a Raspberry Pi or laptop and processes using Google Speech-to-Text to move CAD drawings (and thus potentially robot arms, etc., for delivering material) into desired orientations (Fig. 1 middle). The second system (to be showcased) uses a Sonoff smart-home relay to convert voice commands sent to an Amazon Echo Dot into instructions for a PLC-driven turntable, delivering desired fasteners directly to the operator (Fig. 1 right).

\section{ACKNOWLEDGMENTS}

We thank the Boson One team for doing the first example solution.

\section{REFERENCES}

[1] B. Schönfuß, D. McFarlane, N. Athanassopoulou, L. Salter, L. de Silva, and S. Ratchev. 2020. Prioritising Low Cost Digital Solutions Required by Manufacturing SMEs: A Shoestring Approach. In SOHOMA. Springer, 290-300. 